\title{
The Precise Vehicle Retrieval in Traffic Surveillance with Deep Convolutional Neural Networks
}

\author{
Boyang Su, Jie Shao, Jianying Zhou, Xiaoteng Zhang, Lin Mei, and Chuanping $\mathrm{Hu}$
}

\begin{abstract}
This paper describes a system for precise vehicle retrieval in traffic surveillance, exploiting image representation based on Convolutional Neural Network. Our training data contains about $450 \mathrm{k}$ vehicle face images cropped from different scene and angle. We prepare two test datasets, one set of images all from daytime and another set of images in night scene. Experiment results on data from a real city traffic surveillance network validate and evaluate our system. We demonstrate that our CNN features have outstanding performance in both two test-sets and achieve an expected recall of $97.89 \%$ and precision of $58.22 \%$ at top 100 for the first test-set and recall of $97.09 \%$ and precision $57.57 \%$ for the second one. Our work is very import especially in traffic surveillance and public surveillance to find one special vehicle.
\end{abstract}

Index Terms-Precise vehicle retrieval, convolutional neural network, siamese structure.

\section{INTRODUCTION}

The number of vehicles grows rapidly in cities, and the traffic surveillance system usually has tens of thousands of cameras connected into a huge network. Vehicle retrieval has received much attention in recent years. It is an important part of intelligent transportation system (ITS) or Smart City. However, it is a great challenge task due to the following difficulties.

1) LPR (License Plate Recognition) is a common solution for vehicle retrieval problem. However, LPR is incompetent on the situation of blurred-license cars, unlicensed and fake-license cars.

2) This task is on the key work "precise", which means only the exactly same car can be identified as true positives. Others are negatives even the vehicle brand is same, which always look very similar to the query and cannot be solved by traditional methods.

3) Both vehicle brand and other information (interior trim, annual inspection tags and drivers, etc.) should be considered. But it's very hard to make a common model because it is always personalized.

In computer vision field, visual image retrieval is a classical problem. It is a process of browsing, searching and retrieving images from a big image database. Image retrieval returns a list of images with the same class as query image in accordance with a given retrieval standard. Traditional and common methods of visual image retrieval utilize key representations such as texts [1], colors [2], shapes and

Manuscript received December 22, 2015; revised March 12, 2016. This work was supported in part by the Third Research Institute of Ministry of Public Security.

The authors are with the Internet of Things Technology Department, the Third Research Institute of the Ministry of Public Security, Shanghai, P.R. China (e-mail: uestcsby@163.com). textures [3] to describe images and obtain certain achievements in early years.

Our retrieval task is different from the common retrieval work, which is known as a 'fuzzy' retrieval. As we mentioned above, vehicle retrieval is on the key word "precise", only the exactly same car can be identified as true positives. Moreover, traditional CBIR method cannot meet the requirements of 'precise' in such a mass data today, so we seek a more powerful method to solve the vehicle retrieval problem.

Deep convolutional neural network have achieved a great progress in the field of computer vision, and other fields. Various work on convolutional neural networks (CNN) (Simonyan et al., 2013 [4], Girshich et al., 2014 [5], Chatfield et al., 2014 [6], Zeiler \& Fergus, 2014 [7]) have successfully demonstrated its powerful representation on visual recognition. For the visual image retrieval task, the results of researches began to announce recent two years.

Since CNN features have been proved to be effective as a state-of-the-art descriptor, we choose $\mathrm{CNN}$ to solve the vehicle retrieval problems. CNN feature can overcome the deficiencies mentioned above. Besides, personalized features of each vehicle such as interior trim, annual inspection tags and drivers can be captured and utilized to perform a diversified retrieval.

In 2005, LeCun et al., [8], [9] published a new loss function named "contrastive loss function" in Siamese network. Siamese network is a similar with $\mathrm{CNN}$, with a contrastive loss function to $\mathrm{CNN}$, usually was used as verification task, such as face verification or signature verification. Recently, a lot of researchers and facilities, such as Xiaogang Wang [10], Stan Z. Li [11], Facebook [12] and Google [13] etc., used Siamese network or similar with contrastive loss function in networks for face representation system and achieved amazing results. Inspired by these works, we transplant the Siamese structure to our retrieval system for feature generation.

In this paper, we focus on the targeted traffic images retrieval, which is a very important part of intelligent transportation system (ITS) or Smart City. Our goal is to perform a precise retrieval which can find "the" car rather than "a" same car. In order to generate a robust and efficient representation to distinguish images and locate the right target, we build several powerful convolutional neural networks. Among these models, network with 'Siamese' architecture achieves the best descriptors. Our model performance is measured by precision, recall and AP of top $\mathrm{N}$ images on the rank list, and our system is validated and evaluated by the experiment results on the data which is from a real city traffic surveillance network. As the experimental results shown below, our system is robust 
enough on deformation and aberration, even on a change of license plate or car trim.

\section{RELATED WORK}

\section{A. Content-Based Image Retrieval}

Content-based image retrieval (CBIR) is one of the fundamental research extensively studied in computer vision community for decades. CBIR aims to search for images by analyzing their visual contents. Variety of low level feature descriptors have been proposed for image representation such as SIFT [14]. Conventional CBIR approaches usually choose rigid distance functions such as Euclidean distance or cosine similarity on some extracted low-level features.

In CBIR, the retrievals are based on matching the features of the query image to image database through some feature similarity estimation. So the selected features of images and the pattern are crucial to the image retrieval. Broadly defined, the feature of an image includes textual feature and visual feature can be distinguished as primitive feature and domain-specific feature. The former, includes color, shape and texture, represents the common features of images while the latter includes face recognition and finger prints, which form a sort of high level image description or meta-object.

As a research hot field CBIR technique has achieved great improvement, but this computer-centric query is not competent to fully satisfy the need of the researchers, since the objective description of images. This is reflected in the subjectivity and differential of human perception, and the disconnection between visual features and high-level semantic.

\section{B. Deep Learning}

Deep learning refers to a class of machine learning techniques. In deep learning methods, layers of information processing stages in hierarchical architectures.

Deep learning has a long history, and its basic concept is from artificial neural network research. Feed-forward neural networks with many hidden layers are a good example of deep architecture. Back-propagation, popularized in 1980's, has been a well-known algorithm for learning the weights of these networks. For example, LeCun et al. [15] successfully adopt the deep supervised back-propagation convolutional network for digit recognition. The deep convolutional neural networks (CNNs) proposed in [16] came out first in the image classification task of ILSVRC-2012. The model was trained on more than one million images, and has achieved a winning top-5 test error rate of $15.3 \%$ over 1,000 classes.

After that, some recent works get better results by improving $\mathrm{CNN}$ models. The top-5 test error rate decreased to $6.67 \%$ in [17] by training a 22 layers deep networks. Besides image classification, the object detection task also benefits from the CNN model, as reported in [18].

Some researchers attempt to address the long-standing fundamental feature representation problem in CBIR by using deep learning methods [19]. They investigate a framework of deep learning with application to CBIR tasks with an extensive set of empirical studies by examining a state-of-the-art deep learning method (convolutional neural networks).

\section{PROPOSED METHOD}

CNN has been proved to be an outstanding solution for classification task due to its strong descriptive power, hence we try to utilize CNN to solve image retrieval problems. In our early work, models were trained with ImageNet using the framework of AlexNet, and fine-tuned with our vehicle datasets. Then we simply extract the middle layer features as the vehicle representation feature vector and acquire a retrieval result by computing the distance of feature vectors of two images. The CNN model leads to breakthrough in retrieval performance, however it is still unsatisfied with our practical need. With the development of our studies on model frameworks, we believed a change of our model in framework can lead the way out.

\section{A. CNN-P as Brand Classification}

Our first try is to train a classification model using famous AlexNet framework. The retrieval performance is significantly better than the traditional methods. We then try more sophisticated models to realize the recent advances from deep learning communities.

The model which achieves good retrieval results is a CNN classification model called CNN-P model ( $\mathrm{P}$ means precise). We remove one fully-connected layer and change the filter amount. It is constructed by combining many tricks from recent successful networks including small filter and very deep architecture [20].

The proposed network includes 10 convolutional layer, 5 pooling layers and 1 fully connected layers, the detailed architecture of which is shown in Table I. The size of all filters in the network is $3 \times 3$ that equals to the setting of [16], which achieved high performance in ImageNet 2014 challenges by a 19 layer network using small filter. We use multiple small filters to approximate large filters and remove redundant fully-connected layer to reduce the number of parameters.

The comparing structure is shown below in Table I. CNN-P is a classification model trained by more than 750kinds of vehicle brands. The division of classes is based on the type amount of the vehicles. We use 460k images to train this model, and each class contains at least $3 \mathrm{k}$ images.

The experiment result, in Table II, shows that this model is excellent for classification but still insufficient for retrieval. Images in the rank list looked the same, but actually the licenses are quite different. We believed that the description ability of the model was limited by the $750+$ classes, and the personalized information worked unobvious. Hence, we adopt a new label discrimination standard to overcome this problem.

\section{B. CNN-P as Vehicle Verification}

As mentioned above, the labels restrict the performance of the retrieval system. Our retrieval task can be considered as an intra-class retrieval, while classification can only maximize the inter-class distance. So a manual classification is operated on our database and more than $10 \mathrm{~K}$ car images are collected to compose a new training set. There are about 45 images for each label, hence we have more than $450 \mathrm{k}$ images in training set in total, almost same as our classification dataset. Through this method, we try to eliminate the inter-class interference artificially. 
We set labels as the vehicle plates instead of their brands inspired by the face verification [11]. This label pattern satisfies our need of retrieval, since we define vehicle images with the same plate as the 'same'. We believe that vehicle verification is the right formulation for vehicle retrieval. Deep model trained on this dataset can learn efficient representation for vehicle retrieval.

We still use CNN-P as our classification model. Network configuration was exactly same as brands classification.

TABLE I: OUR MODEL (CNN-P) AND ALEXNET (CNN-A)

\begin{tabular}{c|c|c}
\hline \hline \multirow{2}{*}{ Layer } & \multicolumn{2}{|c}{ Filter Size/Stride } \\
\cline { 2 - 3 } & CNN-P & CNN-A \\
\hline conv1-1 & $64 \times 3 \times 3 / 1$ & $96 \times 11 \times 11 / 4$ \\
\hline ponv1-2 & $64 \times 3 \times 3 / 1$ & $3 \times 3 / 2$ \\
\hline conv2-1 & $2 \times 2 / 2$ & $256 \times 5 \times 5 / 1$ \\
conv2-2 & $128 \times 3 \times 3 / 1$ & $3 \times 3 / 2$ \\
\hline pool2 & $2 \times 2 / 2$ & $384 \times 3 \times 3 / 1$ \\
\hline conv3-1 & $96 \times 3 \times 3 / 1$ & - \\
\hline conv3-2 & $192 \times 3 \times 3 / 1$ & $384 \times 3 \times 3 / 1$ \\
\hline pool3 & $2 \times 2 / 2$ & - \\
\hline conv4-1 & $128 \times 3 \times 3 / 1$ & $256 \times 3 \times 3 / 1$ \\
conv4-2 & $258 \times 3 \times 3 / 1$ & $3 \times 3 / 2$ \\
\hline pool4 & $2 \times 2 / 2$ & 4096 \\
\hline conv5-1 & $160 \times 3 \times 3 / 1$ & 0.5 \\
\hline conv5-2 & $640 \times 3 \times 3 / 2$ & 4096 \\
\hline pool5 & $7 \times 7 / 1$ & 0.5 \\
\hline fc1 & 10957 &
\end{tabular}

\section{CNN-P with Siamese}

Based on the CNN-P, we import the Siamese structure. The architecture of our Siamese network is summarized in Table I. It contains five convolutional layers and one fullconnected layer. Two loss functions, a softmax loss function and a contrastive loss function, were used at the same time in the network. A contrastive loss function is employed to learn the parameters, in such a way that neighbors are pulled together and non-neighbors are pushed apart. As in [9], contrastive loss function is given by the expression:

$$
\begin{gathered}
L(W)=\sum_{i=1}^{P} L\left(W,\left(Y, X_{1}, X_{2}\right)^{i}\right) \\
L\left(W,\left(Y, X_{1}, X_{2}\right)^{i}\right)=(1-Y) L_{G}\left(E_{W}\left(X_{1}, X_{2}\right)^{i}\right)+ \\
Y L_{l}\left(E_{W}\left(X_{1}, X_{2}\right)^{i}\right)
\end{gathered}
$$

where $\left(Y, X_{1}, X_{2}\right)^{I}$ is the $i$-th sample, which is composed of a pair of images and a label (genuine or impostor), $L_{G}$ is the partial loss function for a genuine pair, $L_{I}$ the partial loss function for an impostor pair, and $P$ the number of training samples.

Samples with the same label are defined as a genuine pair in the contrastive loss, otherwise as an imposter pair. As in other CNN networks for classification, the softmax loss function followed the fully connected layer, but contrastive loss function followed the last pooling layer as the same in [11]. A comparison of AlexNet structure and our model is shown in Table I.

The framework of our network is similar with the structure in [11]. But as we are dealing with totally different data, in our model the input image size is $224 \times 224 \times 3$, cropped from $256 \times 256$ color images. The same as in face verification problems, two cars with the same vehicle license plate are verified as the similar pair else as different non-matching pair. Alignment or mirror operation is unnecessary in our model. The difference between the CNN framework and ours is the number of the filter using in the first convolutional operation (64 filters but not 32), and before the last pooling 640 dimension features is generated. The stride in convolutional operation is 2 . In the training, the learning rate was initialized at 0.001 and reduced two times prior to termination. Totally, we trained the Siamese network for about 9 epochs through the training sets before the optimization done. The CNN framework is shown in Fig. 1.

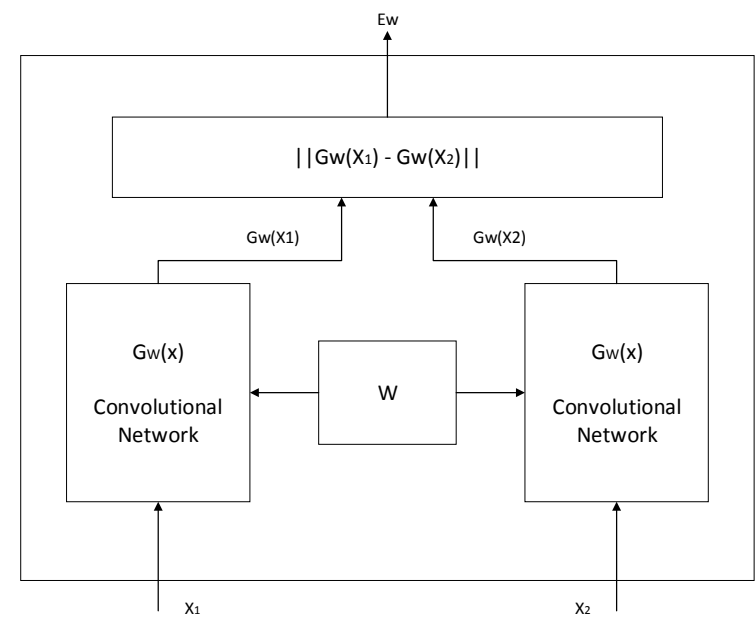

Fig. 1. Siamese architecture.

\section{CNN-P for Vehicle Retrieval}

Visual image feature generated by CNN models above, such as pool5 or fc1, can be considered as high dimensional feature vector in the process of measuring similarity. Therefore, we can regard image feature as a point in the ndimensional vector space based on Vector Space Model, then describe the similarity of two vectors by computing their distance. Traditional methods for computing distance are L1 and L2 norm, Euclidian distance, Mahalanobis distance, histogram intersection and cosine similarity. In retrieval works, Euclidian distance is usually the first choice as distance measurement, however, in this paper, we adopt cosine similarity as our measurement of similarity. Euclidian is the absolute distance of the spatial feature points, which is directly related with the points coordinates. While cosine similarity measures the angles of space vectors. It focuses on the differences in direction, not position. In Fig. 2 we show the characteristics of two measurements. Therefore, cosine similarity has a strong robust on all kinds of affine transformations as well as partial shelter or missing.

The features from both fully-connected layer (fc1) and last pooling layer (pool5) are used for feature representation. Compared with the last pooling layer (pool5) in the performance of image descriptive power, we conclude that: 1) pool5 is a set of responsible node to the filters and retains location information, while fc are encoded features with semantic description. 2) Spatial features such as pool5 cannot describe images independently: pool5 represent meaningless shapes or textures that all vehicles exist. 3) Encoded features are higher level of descriptors: fc1 
demonstrates that combination of spatial features with certain logistic rules can distinguish objects.

We use three judgment criteria for retrieval performance: recall (measurement of the ability of a system that can retrieve relevant information), precision (evaluation of the capacity of a system that can reject uncorrelated information) and AP (average precision). Recall and precision have opposite interdependent relationship, that means a raising recall will reduce precision, and vice versa.
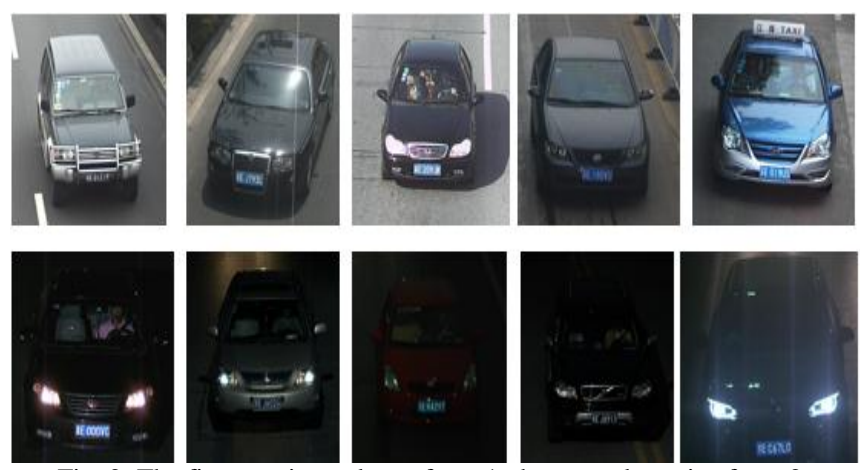

Fig. 2. The first row is a subset of test 1 , the second row is of test 2.

\section{EXPERIENCE RESULT}

To evaluate the retrieval result, we use two networks in the experiment. AlexNet [20] that available on Krizhevsky's homepage. This network is kept intact to produce a benchmark for the evaluation of retrieval. Then we generate our network CNN-P and trained on both brand classification and vehicle verification, and the model with "Siamses" structure.

\section{A. Preparion for Test-Data}

We build two test-groups for this test. In each test 25 query images are picked from our database, each query image has more than 50 matching pairs. The difference between two tests is that query images in test-1 are composed of daytime images, while queries in test- 2 are from night scene. Test- 2 is more challenge than test- 1 . We select very common vehicles in tests in order to check whether CNN-P can extract features in high degree of distinction. Samples are shown in Fig. 3.

\section{B. Vehicle Retrieval on Brands Classification Models}

TABLE II: RETRIEVAL RESUltS OF Two MODELS TRAINED ON BRANDS CLASSIFICATION

\begin{tabular}{c|c|c|c|c}
\hline Model & $\mathrm{R} @ \mathrm{~K}=50$ & $\mathrm{P} @ \mathrm{~K}=50$ & $\mathrm{R} @ \mathrm{~K}=100$ & $\mathrm{P} @ \mathrm{~K}=100$ \\
\hline CNN-A & 63.6 & 63.23 & 82.92 & 45.22 \\
\hline CNN-P & $\mathbf{6 9 . 7 0}$ & $\mathbf{7 9 . 5 2}$ & $\mathbf{8 8 . 4 6}$ & $\mathbf{5 2 . 0 8}$ \\
\hline
\end{tabular}

We compare the AlexNet fine-tuned with $750+$ classes as well as CNN-P, the result is shown in Table II. The difference of this two model is the parameter amount of the filters and the depth. $\mathrm{R}$ means recall, and $\mathrm{P}$ means precision. $\mathrm{K}$ represent the number we check from rank results. There are 5\% improvement on $\mathrm{R} @ \mathrm{~K}=100$, and 6\% on $\mathrm{P} @ \mathrm{~K}=100$. The result demonstrates the structure of CNN-P is more powerful and competent to our task. Results show that with much powerful model the performance is improved greatly.

\section{Experiment on Vehicle Verification}

In this part, we train $\mathrm{CNN}-\mathrm{P}$ with different training set. A significant increase (up to $6 \%$ improvement on $\mathrm{R} @ \mathrm{~K}=100$ ) arises by adopting model from trained on brands classification to vehicle verification. That means the classification by individual vehicle can help extract pivotal and useful information which is needed in retrieval work, especially include interior trim, annual inspection tags and drivers, etc. (See Table III).

TABLE III: PERFormance CoMPaRISON OF OUR MOdEl TRAINED By BRANDS CLASSIFICATION (CNN-P) AND VEHICLE VERIFICATION (CNN-P*)

\begin{tabular}{c|c|c|c|c} 
Model & $\mathrm{R} @ \mathrm{~K}=50$ & $\mathrm{P} @ \mathrm{~K}=50$ & $\mathrm{R} @ \mathrm{~K}=100$ & $\mathrm{P} @ \mathrm{~K}=100$ \\
\hline CNN-P & 69.70 & 79.52 & 88.46 & 52.08 \\
\hline $\mathrm{CNN}^{*}{ }^{*}$ & $\mathbf{8 0 . 7 3}$ & $\mathbf{9 2 . 0 2}$ & $\mathbf{9 4 . 9 5}$ & $\mathbf{5 6 . 1 0}$ \\
\hline
\end{tabular}

\section{Test of Performance}

A comprehensive experiment is operated to verify the effect of Siamese architecture $(\mathrm{CNN}-\mathrm{P} * \mathrm{~S})$. We show the retrieval result in Table IV. Test- 1 contains a group of query images from daytime, while test 2 are images from night scene, and they share the same database. We show our retrieval result of features from pool5 and fc1. Another performance argument $\mathrm{AP}$ is presented as a judgment to $\mathrm{CNN}-\mathrm{P} * \mathrm{~S}$, this argument can verify the top rate of retrieval results.

TABLE IV: TwO TESTS ON CNN-P* AND CNN-P*S (CNN-P* WITH SIAMESE)

\begin{tabular}{|c|c|c|c|c|c|c|c|c|}
\hline Model & & Feature & $2 \mathrm{R} @ \mathrm{~K}=50$ & $\mathrm{R} @ \mathrm{~K}=100$ & $\mathrm{P} @ \mathrm{~K}=50$ & $\mathrm{P} @ \mathrm{~K}=100$ & $\overline{\mathrm{AP} @ \mathrm{~K}=50}$ & AP@K=100 \\
\hline \multirow{2}{*}{ CNN-P* } & \multirow{4}{*}{$\begin{array}{c}\text { Test- } \\
1\end{array}$} & poo5 & 69.91 & 85.38 & 79.81 & 50.26 & 66.80 & 78.07 \\
\hline & & $\mathrm{fc} 1$ & 80.73 & 94.95 & 92.02 & 56.10 & 79.43 & 91.53 \\
\hline \multirow{2}{*}{$\begin{array}{l}\mathrm{CNN}- \\
\mathrm{P} * \mathrm{~S}\end{array}$} & & pool5 & 74.62 & 88.87 & 85.17 & 52.49 & 72.82 & 84.11 \\
\hline & & fc1 1 & 84.09 & 97.89 & 95.92 & 58.22 & 83.37 & 96.17 \\
\hline \multirow{2}{*}{ CNN-P* } & \multirow{4}{*}{$\begin{array}{c}\text { Test- } \\
2\end{array}$} & pool5 & 51.35 & 67.30 & 58.85 & 39.47 & 44.69 & 53.76 \\
\hline & & $\mathrm{fc} 1$ & 71.27 & 88.18 & 81.50 & 52.04 & 67.37 & 79.84 \\
\hline \multirow{2}{*}{$\begin{array}{c}\mathrm{CNN}- \\
\mathrm{P} * \mathrm{~S}\end{array}$} & & pool5 & 58.04 & 73.69 & 67.34 & 43.77 & 53.31 & 63.48 \\
\hline & & $\mathrm{fc} 1$ & 82.95 & 97.09 & 94.54 & 57.57 & 81.84 & 94.54 \\
\hline
\end{tabular}

With Siamese architecture, we obtain top points among all results, and successfully cut the error rate down to the range of $3 \%$. We set another test to check the robustness of $\mathrm{CNN}-\mathrm{P} * \mathrm{~S}$. There is only less than $1 \%$ drop on the 
performance. The exciting result demonstrates $\mathrm{CNN}-\mathrm{P} * \mathrm{~S}$ have outstanding robustness on illumination. In both tests, the AP (average precision) is very high, that means our model can work stably in different situations and the target images in rank list are in very front.

We show examples of correct results and faults result in Fig. 3. The correct results demonstrate the robustness of our model in scale, rotation and illumination. The fault ones have the same brands with query image, hence the similarity is high. In normal retrieval cases the fault results can be considered as true, however to our task, these two vehicles are identified as different images.

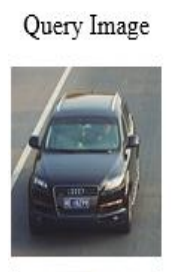

Correct Retrieval Samples

Fault Retrieval Samples
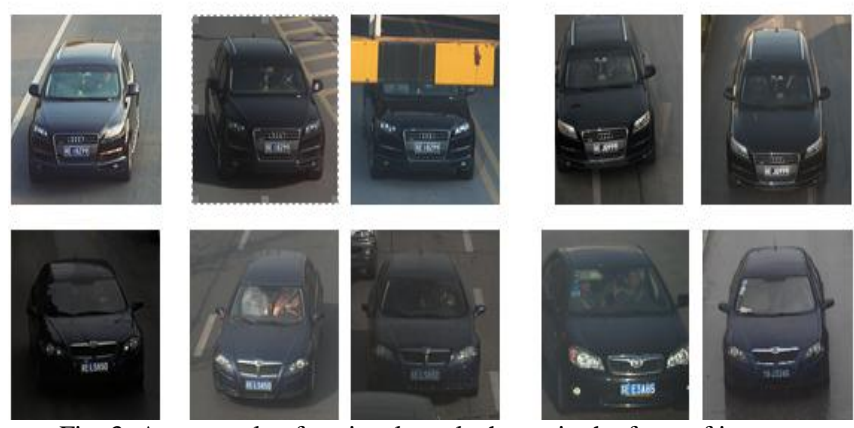

Fig. 3. An example of retrieval result shown in the form of images.

\section{CONCLUSION}

This paper shows our study on precise vehicle retrieval, and demonstrates that precise vehicle retrieval is a challenge problem due to its target which should be "the" car rather than "a" same car. Powerful deep learning model can achieve better performance than traditional hand-craft feature descriptors, even when we use the "wrong" formulation (trained by brands classification). Vehicle verification can tell the subtle difference between the different car with same brands and color. Performance can be further boosted by using Siamese architecture even using night query images.

Through our study, we find that the Siamese network can imitate the behavior of human learning methods like to learn things by comparison. Our rigorous experiment results demonstrate that 1) $\mathrm{CNN}-\mathrm{P} * \mathrm{~S}$ effectively reduces the intraclass distances and expends the inter-class distance. 2)The comparing learning (Siamese structure) is higher level of human learning than memorizing learning which represents the traditional convolutional neural networks, since the retrieval process itself is a kind of comparison. Therefore, the reserved correlative features from matching pairs and uncorrelated features of non-matching pairscan reveal outstanding and efficient descriptive power in retrieval tasks 3) A combination of spatial features and semantic features is a more powerful descriptor than any of them, and qualified with complex retrieval tasks. We believe a more exciting achievement can be obtained by a further study on Siamese architecture in the future.

\section{ACKNOWLEDGEMENT}

This work was supported in part by the National Science and Technology Major Project under Grant 2013ZX01033002-003, in part by the National High
Technology Research and Development Program of China (863 Program) under Grant 2013AA014601, 2013AA014603, in part by National Key Technology Support Program under Grant 2014JSYJB006, and the project of Shanghai Municipal Commission of Economy and Information under Grant 12GA-19.

\section{REFERENCES}

[1] G. Salton and C. Buckley, "Term-weighting approaches in automatic text retrieval," Inf. Process. Manage., vol. 24, no. 5, pp. 513-523, 1988.

[2] M. J. Swain and D. H. Ballard, "Color indexing," International Journal of Computer Vision, vol. 7, no. 1, pp. 11-32, 1991.

[3] D. G. Lowe, "Object recognition from local scale-invariant features," in Proc. the International Conference on Computer Vision, 1999, pp. 1150-1157.

[4] K. Simonyan et al., "Deep inside convolutional networks: Visualising image classification models and saliency maps," 2013.

[5] B. R. Girshick, N. F. Iandola et al., "Deformable part models are convolutional neural networks," CoRR, abs/1409.5403, 2014.

[6] K. Chatfield, K. Simonyan, A. Vedaldi et al., "Return of the devil in the datails: Delving deep into convolutional nets," CoRR, 2014.

[7] D. M. Zeiler and R. Fergus, "Visualizing and understanding convolutional networks," ECCV, pp. 818-833, 2014.

[8] S. Chopra, R. Hadsell, and Y. LeCun, "Learning a similarity metric discriminatively, with application to face verification," in Proc. Computer Vision and Pattern Recognition Conference, 2005.

[9] R. Hadsell, S. Chopra and Y. LeCun, "Dimensionality reduction by learning an invariant mapping," in Proc. Computer Vision and Pattern Recognition Conference, 2006.

[10] Y. Sun, Y. Chen, X. Wang, and X. Tang, "Deep learning face representation by joint identification-verification," in Proc. Neural Information Processing Systems Conference, 2014.

[11] Y. Dong, L. Zhen, L. Shengcai, and Z. L. Stan, "Learning face representation from scratch," arXiv preprint arXiv:1411.7923, 2014.

[12] Y. Taigman, M. Yang, and M. A. Ranzato, "Deepface: Closing the gap to human-level performance in face verification," CVPR, 2014.

[13] F. Schroff, D. Kalenichenko, and J. Philbin, "A unified mbedding for face recognition and clustering," arXiv, arXiv: 1503.03832.

[14] D. G. Lowe, "Object recognition from local scale-invariant features," ICCV, pp. 1150-1157, 1999.

[15] Y. LeCun, L. Bottou, Y. Bengio, and P. Haffner, "Gradient-based learning applied to document recognition," Proceedings of the IEEE, vol. 86, no. 11, pp. 2278-2324, 1998.

[16] A. Krizhevsky, I. Sutskever, and G. E. Hinton, "Imagenet classification with deep convolutional neural networks," NIPS, 2012.

[17] C. Szegedy, W. Liu, Y. Jia et al., "Going deeper with convolutions," arXiv preprint arXiv:1409.4842, 2014.

[18] R. B. Girshick, J. Donahue, T. Darrell, and J. Malik, "Rich feature hierarchies for accurate object detection and semantic segmentation," CoRR, abs/1311.2524, 2013.

[19] J. Wan, D. Wang, S. C. H. Hoi et al., "Deep learning for contentbased image retrieval: A comprehensive study," in Proc. the ACM International Conference on Multimedia, ACM, 2014, pp. 157-166.

[20] K. Simonyan and A. Zisserman, "Very deep convolutional networks for large-scale image recognition," arXiv preprint arXiv:1409.1556, 2014.

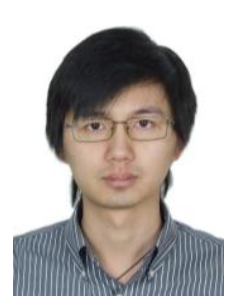

Boyang Su finished his bachelor's degree in University of Electronic and Science of Technology of China in 2010. Then, Boyang got his master's degree from the Rochester Institute of Technology in 2013 with a major of electrical engineering.

Since he graduated from RIT, he has worked in the TRIMPS until now as an algorithm engineer, working on computer vision and machine learning such as image retrieval.

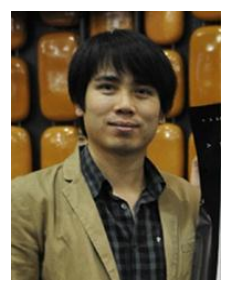

Jie Shao received his master degree from Shanghai Institute of Optics and Fine Mechanics, Chinese Academy of Sciences.

His research interest mainly focused on computer vision, machine learning, especially including deep learning for visual recognition, large scale image retrieval and etc. 


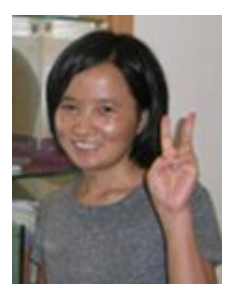

Jianying Zhou received her Ph.D. degree from the Institute of Automation, Chinese Academy of Sciences.

Her research interest covers applications of computer vision, pattern recognition and machine learning. She mainly focused on deep learning for visual recognition, especially including image classification, object detection.

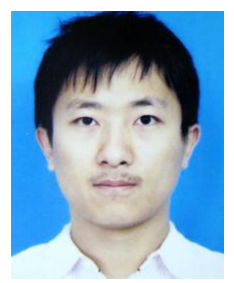

Xiaoteng Zhang received his master degree from the Science of Mathematics College, University of Science and Technology of China.

His research interest covers applications of computer vision, pattern recognition and machine learning. He mainly focused on deep learning for visual recognition, especially including image classification, image localization, object detection.

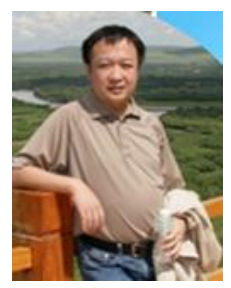

Lin Mei is a director of the center of internet of things in TRIMPS

His research interest covers computer vision, AI, applications of the internet of things, big data etc.

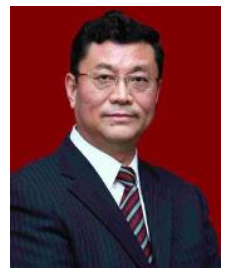

Chuanping $\mathbf{H u}$ is a director of TRIMPS. He dedicated to the high-tech in the field of firefighting equipment flexible control technology, robot technology, satellite emergency communication and command technology, surveillance video structured information description technology and etc. 